\title{
Editorial: Chimera States in Complex Networks
}

\author{
Eckehard Schöll ${ }^{1}$, Anna Zakharova ${ }^{1}$ and Ralph G. Andrzejak ${ }^{2 *}$ \\ ${ }^{1}$ Institut für Theoretische Physik, Technische Universität Berlin, Berlin, Germany, ${ }^{2}$ Department of Information and \\ Communication Technologies, Universitat Pompeu Fabra, Barcelona, Spain
}

Keywords: chimera states, networks, synchronization, multilayer network, complex

\section{Editorial on the Research Topic}

\section{Chimera States in Complex Networks}

Dynamics of complex networks is a central issue in non-linear science with applications to different fields ranging from natural to technological and socio-economic systems. The interplay of non-linear dynamics, network topology, naturally arising delays, and random fluctuations results in a plethora of spatio-temporal synchronization patterns. Chimera states in dynamical networks consist of coexisting domains of spatially coherent (synchronized) and incoherent (desynchronized) behavior. They are a manifestation of spontaneous symmetry-breaking in systems of identical oscillators, and occur in a variety of physical, chemical, biological, neuronal, ecological, technological, or socio-economic systems.

In this Research Topic, we focus on recent developments with future promising perspectives, for instance, chimera patterns in small networks, adaptive networks, complex coupling topologies like modular, fractal, or multilayer connectivity, coupled phase and amplitude dynamics, information flow in chimera states, as well as filtering and control methods for stabilizing chimera states.

In particular, multilayer networks where the nodes are distributed in different layers offer

OPEN ACCESS

Edited and reviewed by: Axel Hutt,

German Weather Service, Germany

*Correspondence: Ralph G. Andrzejak ralph.andrzejak@upf.edu

Specialty section:

This article was submitted to

Dynamical Systems,

a section of the journal

Frontiers in Applied Mathematics and

Statistics

Received: 07 November 2019

Accepted: 15 November 2019

Published: 29 November 2019

Citation:

Schöll E, Zakharova A and Andrzejak RG (2019) Editorial:

Chimera States in Complex Networks. Front. Appl. Math. Stat. 5:62. doi: 10.3389/fams.2019.00062 better representation of the topology and dynamics of real-world systems in comparison with one-layer structures. One of the most promising applications of the multilayer approach is the study of the brain, where the neurons can form different layers depending on their connectivity through chemical or electrical synapses, or technological interdependent systems, i.e., those systems in which the correct functioning of one of them strongly depends on the status of the others. For instance, multilayer networks with interconnected layers naturally occur in transportation systems and electrical power grids. The intriguing dynamics of multiplex networks includes relay synchronization and partial synchronization patterns like chimera states.

The articles in this Research Topic are grouped such that we start from theoretical aspects and methods, and then proceed to various applications in physics, chemistry, neurosciences, ecology, and social dynamics. The first six articles focus on theoretical advances. Deschle et al. quantify the directed flow of information within and between two coupled subpopulations of a phase oscillator network in a chimera state. For this purpose they apply the delayed mutual information to the time points at which the individual phases pass through their respective Poincaré sections. The authors report on a functional flow of information from the desynchronized to the synchronized subpopulation of the phase oscillator network. Banerjee et al. study amplitude chimeras, which are distinct from conventional phase chimeras, since the coexistence of coherent and incoherent domains here refers not to the correlation of the oscillator phases, but of their amplitudes: all the oscillators have the same phase velocity, however, the oscillators in the incoherent domain show periodic oscillations with randomly shifted origin. The authors investigate the effect of local low-pass or all-pass filtering in the coupling path, and show that filtering can suppress amplitude chimeras and give rise to global synchrony, in a generic model of Stuart-Landau oscillators. 
The paper by Sathiyadevi et al. investigates imperfect amplitude mediated chimeras and shows that these states occur due to competing attractive and repulsive interactions in non-locally coupled networks of Stuart-Landau oscillators. The distinctive feature of these patterns is that the oscillators constituting the synchronized and desynchronized groups drift randomly in time between the homogeneous and inhomogeneous regimes. The authors demonstrate the robustness of the observed amplitude mediated chimera state against Gaussian white noise. Dmitrichev et al. study the mechanism of chimera state cloning in a large two-layer multiplex network of coupled relaxation oscillators with short-term interactions. In more detail, for certain values of strength and time of multiplex coupling, in the initially disordered layer, the authors detect a chimera state with the same characteristics, like the chimera which has been set in the other layer. The authors show that the cloning is not related to synchronization, but arises from the competition of oscillations in pairs of elements from different layers. Sawicki et al. explore chimera states in a three-layer network of FitzHughNagumo oscillators, where each layer represents a non-locally coupled ring. In particular, the role of time delays introduced in both inter- and intra-layer interactions is investigated. In the parameter plane of the two delay times, the authors determine the regions where chimera patterns occur, alternating with regimes of coherent states. It turns out that a proper choice of time delay allows for achieving the desired state of the network: chimera states or coherent patterns, full or relay inter-layer synchronization. The work of Omelchenko et al. deals with the control of chimera states in multilayer networks. In smallsize networks, chimera states usually exhibit short lifetimes and erratic drifting of the spatial position of the incoherent domain. A tweezer control scheme combining both symmetric and asymmetric feedback loops can stabilize and fix the position of chimera states. Here this control method is applied to a twolayer network of Van der Pol oscillators. Tweezer control, applied to only one layer, successfully stabilizes chimera patterns in the other, uncontrolled layer.

The next seven articles feature various applications. Rode et al. apply chimera states to chemical oscillators combining numerical simulations with experiments on photo-coupled relaxation oscillators. Focusing on the case of strong interactions the authors find in chemical experiments that beyond weak coupling chimera patterns consist of different coexisting cluster states. Moreover, numerical modeling reveals that the observed cluster states result from a phase-dependent excitability that is also commonly observed in neural tissue and cardiac pacemaker cells. Ocampo-Espindola et al. investigate the formation of weak chimera states in modular networks of electrochemical oscillators. Two globally coupled populations of highly nonlinear oscillators which are weakly coupled through a collective resistance are studied experimentally and numerically. The authors find very robust chimera states and conclude that they could provide a mechanism for the generation of chimeras in biological systems. The modular multilayer topology of the connectome of $C$. elegans is studied by Pournaki et al. They consider a network consisting of two synaptic (electrical and chemical) layers and one extrasynaptic (wireless) layer. Synchronization patterns and chimera-like states are investigated using metrics of synchronization based on Euclidean distances and a new method of finding clustered nodes by correlating their dynamical variables. Their findings allow them to relate the dynamics of the model neurons to biological neuronal functions such as motor activities. Argyropoulos and Provata apply chimera states to neural networks of coupled Leaky Integrateand-Fire models. They investigate the formation of chimera states in 2D lattices with hierarchical (fractal) connectivity that has the form of a deterministic or a random Sierpinski carpet. Their findings confirm previous studies on symmetric deterministic hierarchical connectivities and extend them to slanted and random fractals. Hizanidis et al. analyze chimera states in network models of locally and non-locally coupled superconducting quantum interference devices (SQUIDs). They demonstrate numerically that for both types of coupling, chimera states as well as other spatially non-uniform states can be generated under time-dependent applied magnetic flux for appropriately chosen initial conditions. Furthermore, chimera states can be achieved in the presence of a constant (DC) flux gradient with the SQUID meta-material initially at rest. Saha et al. apply chimera states to an ecological network. The population dynamics of different species, dispersed in patches and connected by weighted mean-field diffusion, is described by a modified Rosenzweig-MacArthur predator-prey model. Various complex population patterns emerge, namely multicluster and chimera states, besides synchrony and homogeneous steady states. Lugo et al. study chimera states in learning dynamics. An agentbased two-layer model of the social dynamics in which the state of the agents corresponds to one of two possible strategies is used to find new configurations which do not exist in a single isolated social network. They include chimera states in which two different collective states coexist in the network, namely, one with full coordination and one with coexistence of strategies.

\section{AUTHOR CONTRIBUTIONS}

This editorial was written by ES, AZ, and RA.

Conflict of Interest: The authors declare that the research was conducted in the absence of any commercial or financial relationships that could be construed as a potential conflict of interest.

Copyright (c) 2019 Schöll, Zakharova and Andrzejak. This is an open-access article distributed under the terms of the Creative Commons Attribution License (CC BY). The use, distribution or reproduction in other forums is permitted, provided the original author(s) and the copyright owner(s) are credited and that the original publication in this journal is cited, in accordance with accepted academic practice. No use, distribution or reproduction is permitted which does not comply with these terms. 